\title{
Time to Negativity and Factors Associated with Persistent Reverse Transcription-Polymerase Chain Reaction Positivity among Asymptomatic and Mildly Symptomatic Covid-19 Patients
}

\author{
Anitha Abraham ${ }^{1}$, Adarsh M.B. ${ }^{2}$, Kavitha Padinjath ${ }^{3}$, Meera Mini Nandakumar ${ }^{4}$, \\ Rajendra Pilankatta ${ }^{5}$, Raman Swathy Vaman ${ }^{6}$
}

\begin{abstract}
${ }^{1}$ Department of Community Medicine, Government Medical College, Thiruvananthapuram, Previous Government Medical College, Kasaragod, Kerala, India, ${ }^{2}$ Department of Internal Medicine, Government Medical College, Kasaragod, Kerala, India, ${ }^{3}$ Department of Anaesthesia, Government Medical College, Kottayam, Government Medical College, Kasaragod, Kerala, India, ${ }^{4}$ Department of ENT, Government Medical College, Kasaragod, Kerala, India, ${ }^{5}$ Department of Biochemistry and Molecular Biology, Central University of Kerala, Kasaragod, Kerala, India, ${ }^{6}$ Government Medical College, Kasaragod, National Health Mission, Kasaragod, Kerala, India.
\end{abstract}

\section{ABSTRACT}

\section{BACKGROUND}

The Covid-19 infection caused by SARS-CoV-2 has spread rapidly across the world \& has become a global public health emergency. Infected patients tend to carry the virus for a longer period of time, which requires an early isolation and monitoring. Information on persistent RT-PCR positivity and time to negativity are lacking in our population. We wanted to assess the prevalence of persistent RT-PCR positivity, time to a negative test and factors related to it in our population.

\section{METHODS}

This is a retrospective medical record based observational study conducted among lab confirmed and admitted Covid-19 patients. After admission, repeat oropharyngeal swabs were taken at specific time intervals that varied from 3 - 5 days. A persistent RT-PCR positivity was defined as more than 3 repeat positive RT-PCR tests in a patient.

\section{RESULTS}

118 Covid-19 positive patients were included in the study. Mean age was 40.58 yrs. (SD, 11.9 years). Median number of repeat RT-PCR tests was 3 (IQR, 2 - 4 repeats). Persistent RT-PCR positivity was seen in 15 (12.7\%) subjects. Median time taken to first negative RT-PCR test was 9 days (IQR, 6.7 - 13.25 days) and median duration of hospital stay of subjects was 14 (IQR, $10-18$ ). Presence of hypertension, use of antibiotic azithromycin and use of angiotensin receptor blockers were found to be significant risk factors for persistent positivity.

\section{CONCLUSIONS}

Persistent RT-PCR positivity indicating prolonged viral shedding was seen in $12.7 \%$ of patients. Hypertension and use of antibiotic azithromycin were independent risk factors of persistent RT-PCR positivity. The epidemiological burden of the finding needs to be assessed in a wider population with a larger sample size.

\section{KEY WORDS}

COVID-19, RT-PCR, Viral Persistence, Infectivity, Time to Negativity
Corresponding Author: Dr. Raman Swathy Vaman, Superintendent, Government Medical College, Kasaragod, Ukkinadka, Kasaragod dist., Kerala-671552, India, E-mail: ramanvaathapi@gmail.com

DOI: $10.14260 / \mathrm{jemds} / 2021 / 487$

How to Cite This Article: Abraham A, M. B. Adarsh, Padinjath K, et al. Time to negativity and factors associated with persistent RT-PCR positivity among asymptomatic and mildly symptomatic Covid-19 patients. J Evolution Med Dent Sci 2021;10(31):2373-2377, DOI: $10.14260 / j e m d s / 2021 / 487$

Submission 19-03-2021,

Peer Review 01-06-2021,

Acceptance 07-06-2021,

Published 02-08-2021.

Copyright (C) 2021 Anitha Abraham et al. This is an open access article distributed under Creative Commons Attribution License [Attribution 4.0 International (CC BY 4.0)] 


\section{BACKGROUND}

Coronaviruses are enveloped non-segmented RNA viruses belonging to the family Coronaviridae. The current pandemic is caused by the Novel Coronavirus (SARS-CoV-2). ${ }^{1}$ Infection with SARS-CoV-2 is confirmed by the detection of virus RNA in various body fluids. Polymerase chain reaction (PCR) method is considered as the 'gold standard' for its detection. Nasopharyngeal and oropharyngeal swab tests are used as standard tools for diagnosis of SARS-CoV-2.2 Persistent shedding of SARS-CoV-2 has been reported in respiratory and intestinal samples and body fluids. The estimated time until loss of virus RNA detection ranged from 45.6 days for nasopharyngeal swab samples in mild cases to 48.9 days in severe cases, which was longer than those of SARS-CoV-2 and MERS-CoV.,4 The factors related to persistence of viral RNA in patients with Covid-19 infection are not clear. A longer time interval of RT-PCR positivity was reported in older age group (> 65 yrs.) and in patients on glucocorticoid treatment.5,6 Also viral clearance was longer in patients with chronic co morbidities like cardiovascular diseases, diabetes, cancer etc. ${ }^{7}$ The initial discharge criteria set by Ministry of Health \& Family Welfare, Government of Kerala was after obtaining two negative RT-PCR tests even if asymptomatic. Persistent positivity among asymptomatic and mildly symptomatic patients was a significant issue in this aspect. This was consuming much resource in terms of test equipment and human resources and was prolonging the hospital stay of patients. Available studies have looked into prevalence of RTPCR positivity among patients after discharge. Most studies have looked at the point prevalence after discharge. Information on persistent RT-PCR positivity and time to negativity is lacking in our population. A better knowledge could help in policy making in relation to preventive measures, discharge criteria and patient care. Hence, we assessed the prevalence of persistent RT-PCR positivity, time to a negative test and factors related to it in our population.

\section{METHODS}

This is a retrospective medical record based observational study conducted among lab confirmed Covid-19 patients admitted in Government Medical College, Kasaragod, Kerala, India which is a designated Covid hospital. During the study duration the hospital catered asymptomatic and mildly symptomatic male patients and lab confirmed (RT-PCR) positives with uncomplicated upper respiratory tract infections with nonspecific symptoms like fever, headache, mild cough, sore throat etc.

The diagnosis was made by RT-PCR (RealStar® SARS-CoV2 RT-PCR Kit, Altona diagnostics, Germany) from samples collected by oropharyngeal swabs. After admission, repeat oropharyngeal swabs were taken at specific time intervals that varied from 3 - 5 days and patients were discharged from hospital after obtaining 2 consecutive negative RT-PCR results. Hence patients had to undergo repeated RT-PCR testing till they become negative on two consecutive tests. (That's every 48 hrs apart ideally, but it may have delayed 3 to 5 days based on test result availability).
Data of all patients who were 18 years and above admitted from 1st April 2020 to 1st July 2020 was analysed for the study. Details regarding age, number of RT-PCR tests repeated, duration of hospital stay, clinical history, treatment history and lab parameters were collected from case records on a predesigned proforma. A persistent RT-PCR positivity was defined as more than 3 repeat RT PCR tests in a patient which was the median number of RT-PCR tests in the sample. Gap between each RT-PCR test varied between 3 and 5 days based on the timely availability of test results. Ethical clearance for the study was obtained from Institutional Ethical Committee, Government Medical College, Kannur (IEC No. 1022019 / GMCK dated 17.09.20). A waiver for obtaining patient consent was given as the study involved anonymous retrospective data analysis.

\section{Statistical Analysis}

Data was analysed using SPSS software 22. Categorical variables were expressed using frequencies and continuous variables were expressed as median and interquartile ranges or mean and Standard deviation. Bivariate analysis was done to find out the factors related to persistent RT-PCR positivity. Chi-square test was used to evaluate association between categorical variables and outcome variables. Strength of association between exposure and outcome variables was estimated using odds ratio with $95 \%$ confidence interval. Binary Logistic regression was performed using the factors found to be significant in the bivariate analysis. A P value of $<$ 0.05 was taken as significant level for all calculations.

\section{RESULTS}

Total of 118 Covid-19 positive patients were included in the study. Mean age of study subjects was 40.58 yrs. (SD, 11.9 years) ranged from 18 yrs. to 68 yrs. All were males. Most of them were expatriates from Gulf Cooperation Council countries and were on quarantine on reaching. Among this 70 $(59.3 \%)$ were symptomatic (Table 1$)$. Main symptoms were cough (23.7\%), fever (17.8\%), sore throat (17.8\%) and headache $17(14.4 \%)$ respectively. Of the total, $32(27.1 \%)$ had other co-morbidities. Commonest comorbidity was hypertension $(27.1 \%)$. Other co-morbidities were diabetes mellitus $14(11.9 \%)$, chronic obstructive pulmonary disease (COPD) 2(1.6\%), coronary artery disease (CAD) and asthma $1(0.8 \%)$. Smoking and alcohol use were reported by $18(15.3$ $\%)$ and 7 (5.9\%) subjects respectively. Twenty-three (19.5\%) patients received antibiotic azithromycin and only one person received hydroxychloroquine. Median number of repeat RTPCR tests was 3 (IQR, 2 - 4 repeats) in the study subjects. Number of RT-PCR taken ranged from minimum of 2 to 9 repeats. First RT-PCR repeat was positive in 71(60.2\%) of subjects, 2nd repeat in $39.8 \%$ and $3 r$ in $22.9 \%$. More than three repeat RT-PCR positivity was seen in 15 (12.7\%) subjects (Fig1). Median time taken to first negative RT-PCR test was 9 days (IQR, 6.7 - 13.25 days) from the first positive swab result and ranged from 3 days to maximum of 34 days. Median duration of hospital stay of subjects was 14 days (IQR, $10-18$ ) from the date of admission and maximum days of stay was up to 38 days. 


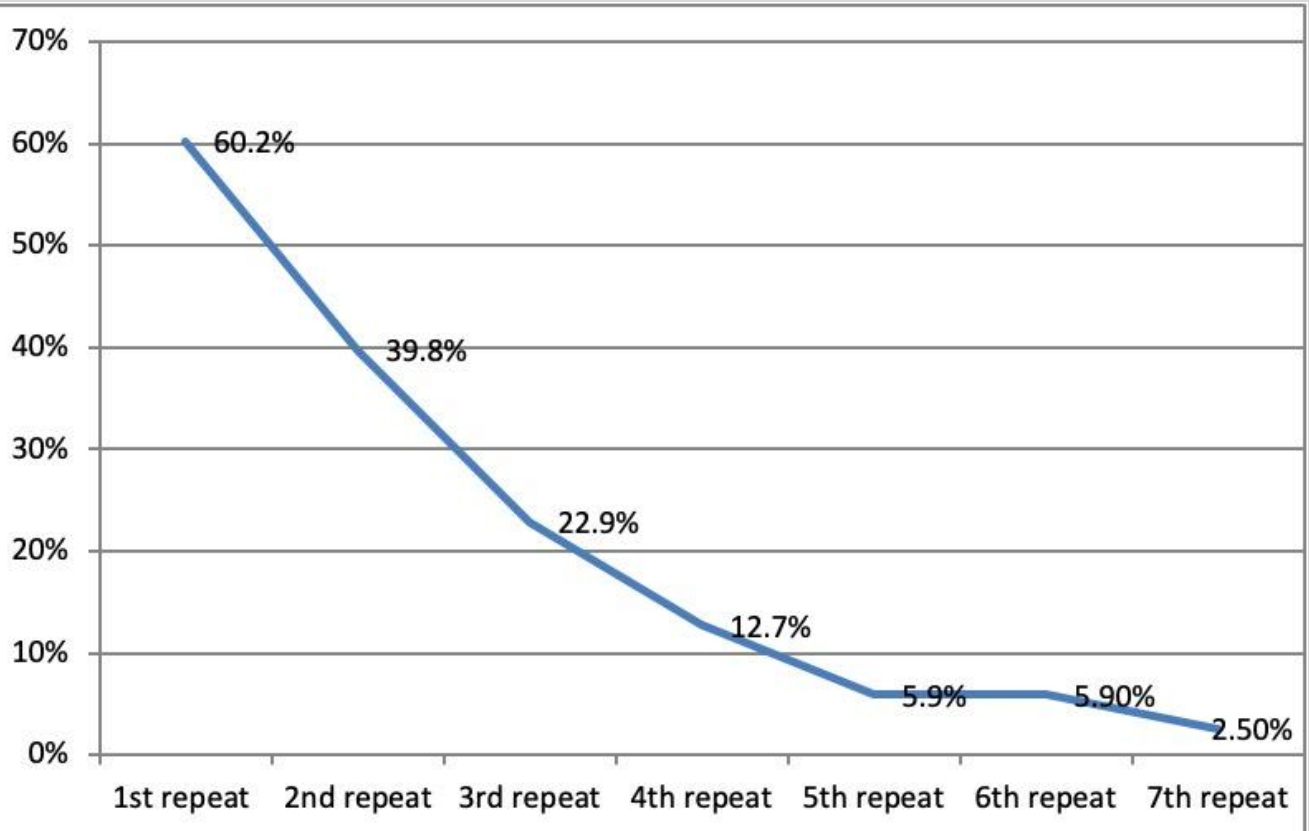

Figure 1. Proportion of Repeated RT-PCR Test Positivity in the Study Subjects

Persistent RT-PCR (more than 3 positive repeats) was seen in $15(12.7 \%)$ out of 118 patients (Table 1$)$. There was no significant difference in mean age of participants across two groups. Proportion of hypertension (53.3 \%) was significantly more in patients with persistent positivity. Similarly, use of antibiotic azithromycin (40.0\%) and angiotensin receptor blockers $(20.0 \%)$ were also significantly associated with persistent positivity. Though the proportion of symptomatic $(73.3 \%)$ and those with co morbidities (40.0\%) were numerically higher in patients with persistent positivity, this was not statistically significant. No significant association was seen with steroid use, presence of diabetes or those who ever smoked (Table1).

On bivariate analysis, presence of hypertension $\{\mathrm{OR}, 5.78$ (95\% CI, 1.8 - 18.07)\}, use of antibiotic azithromycin $\{0 R$, $3.37(95 \% \mathrm{CI}, 1.06-10.72)\}$ and use of angiotensin receptor blockers $\{\mathrm{OR}, 12.62(95 \% \mathrm{CI}, 1.91$ - 83.28)\} were found to be significant risk factors for persistent positivity. On binary logistic regression, adjusted odds for persistent RT-PCR positivity was found significantly higher in patients with hypertension \{Adj OR, 6.36 (95\% CI, 1.93 - 20.95) \} and those treated with drug azithromycin Adj OR, 3.88(95\% CI, 1.1 13.60)\}.

\begin{tabular}{|c|c|c|c|c|}
\hline Characteristics & $\begin{array}{l}\text { All Patients, } \\
\mathrm{N}=118(\%)\end{array}$ & $\begin{array}{l}\text { Persistent } \\
\text { Positivity, N } \\
=15(\%)\end{array}$ & $\begin{array}{c}\text { No Persistent } \\
\text { Positivity, N = } \\
103(\%)\end{array}$ & P Value \\
\hline Mean Age(SD) years & 40.7(SD,11.9) & 38.6(SD,10.8) & 41.01(SD,12.11) & 0.54 \\
\hline Symptomatic & $70(59.3)$ & $11(73.3)$ & $59(57.3)$ & 0.24 \\
\hline $\begin{array}{l}\text { Presence of Co- } \\
\text { morbidities }\end{array}$ & $32(27.1)$ & $6(40.0)$ & $26(25.2)$ & 0.23 \\
\hline Hypertension & $25(21.1)$ & $8(53.3)$ & $17(16.5)$ & 0.001 \\
\hline Diabetes mellitus & $14(11.9)$ & $1(6.7)$ & $13(12.6)$ & 0.54 \\
\hline Azithromycin use & $23(19.5)$ & $6(40.0)$ & $17(16.5)$ & 0.03 \\
\hline $\begin{array}{l}\text { Use of Angiotensin } \\
\text { receptor blockers }\end{array}$ & $5(4.23)$ & $3(20.0)$ & $2(1.9)$ & 0.001 \\
\hline Use of steroids & $4(3.38)$ & $1(6.7)$ & $3(2.9)$ & 0.45 \\
\hline Ever smoker & $18(15.3)$ & $2(13.3)$ & $16(15.5)$ & 0.82 \\
\hline \multicolumn{5}{|c|}{ Table 1. Base Line Characteristics of Study Participants } \\
\hline
\end{tabular}

\section{DISCUSSION}

In this study, among male patients who were asymptomatic or had mild symptoms, persistent RT-PCR positivity was seen in $15(12.7 \%)$ patients. Three $(2.5 \%)$ patients had persistent positivity even on 7 th PCR repeat. The median time to test negativity was 9 days with maximum up to 34 days. Median days of viral shedding varied from 9.5 days to 20 days across studies. $^{8}$ Time to loss of viral RNA detection was estimated to be 45.6 days in nasopharyngeal swabs. ${ }^{3}$ Another study showed RT-PCR positivity in throat samples of patients who recovered from mild disease for up to 50 days. ${ }^{9}$ All these studies looked at a point prevalence after discharge, while we assessed it at regular time intervals. There are no studies that estimated persistent positivity at definite time points.

As per the initial discharge criteria, patients had to be tested negative on two consecutive tests, even if asymptomatic. The need for repeated testing prolonged the duration of hospital stay. In our study, days of hospitalization among patients extended up to 49 days. Even though positive RT-PCR indicates shedding of viral RNA, it detects both viable and dead virus from clinical specimens. Hence it is difficult to say whether patients with persistent positivity are infectious, without a virus isolation or serological investigation. Virological assessment of hospitalized patients showed that, pharyngeal viral shedding was high during the first week of symptoms with peak of RNA concentrations reaching by day 5 and demonstrated a decline by day 10 of onset of symptoms. ${ }^{10}$ Government policy of initial double negative discharge criteria later was changed to single negative test for asymptomatic and mild cases on 10th day of first positive result after reviewing existing evidences from various studies. This reduced burden on health care system \& optimized testing capacity.

Hypertension and antibiotic use were found as independent risk factors for persistent positivity. Hypertension was the most prevalent co-morbidity in our study (27.1 \%). Several other studies also reported 
hypertension as the most common co-morbidity among Covid19 infected patients. ${ }^{11,12}$

Slower viral clearance was associated with co-morbidities such as cardiovascular diseases and diabetes in patients with SARS or influenza A. ${ }^{7}$ Findings from another study suggested that diseases such as hypertension may be associated with disease severity but did not give any relation with duration of viral shedding. ${ }^{13}$ Since it has got an impact on disease outcome, it is important to control blood pressure among Covid-19 patients even if it is not related to duration of viral shedding. Use of angiotensin receptor was more in persistent positivity group, but was not significant in multivariate analysis. Covid patients having hypertension are often treated with ACE inhibitors and angiotensin receptor blockers. Evidences show that SARS-CoV-2 enters cells by binding ACE2 in lungs. ${ }^{14}$ It was found that treatment with these drugs increase ACE2 expression and facilitate viral entry leading to greater lung injury.

On the other hand, ACE inhibitors by reducing angiotensin II formation and ARB blockers by antagonizing angiotensin II action, could reduce the inflammation particularly in lungs, heart and kidneys. There is no evidence yet as to prove that the use of these drugs either improve or worsen the disease and also for discontinuation in management of hypertension in Covid-19 patients. ${ }^{15}$ Use of antibiotic azithromycin was significantly higher in those with persistent positivity. Majority who received azithromycin were symptomatic (87.0 $\%)$ and the chance of viral shedding might be more in them compared to asymptomatic. An open labelled trial showed better viral elimination on adding azithromycin with hydroxychloroquine than HCQ alone. ${ }^{16}$ But the study was limited by small sample size and improper methodology.

In-vitro study in Vero cells added that SARS-CoV replication was not inhibited by azithromycin. Also there was no evidence to support anti-inflammatory effects of drugs in Covid-19 infection. ${ }^{17}$ Use of antibiotics is associated with an alteration in local microbiota in throat. This needs to be looked into as local microbiota acts as a biological barrier for the entry and persistence of pathological microbes in throat and nasopharynx. Older age group was associated with significant prolongation of viral shedding. ${ }^{5}$ But such an association was not seen in our study. Moreover, above 60 population was only $5.9 \%$ and the mean age was comparable in both groups.

The strength of our study was the regular interval sampling that we did.

\section{CONCLUSIONS}

Persistent RT-PCR positivity indicating prolonged viral shedding was seen in $12.7 \%$ of patients. Hypertension and use of antibiotic azithromycin were independent risk factors for persistent RT-PCR positivity. The epidemiological burden of the finding needs to be assessed in a wider population with a larger sample size.

\section{Limitations}

Our study has few limitations. The study population was males who were asymptomatic or had mild symptoms. Although males were known to be affected with severe disease, the effect of sex was not known on viral persistence. The duration of viral shedding may be underestimated in the study as severe cases were not included. As we have not done a viral culture or serology, the clinical significance of the finding needs to be established

Data sharing statement provided by the authors is available with the full text of this article at jemds.com.

Financial or other competing interests: None.

Disclosure forms provided by the authors are available with the full text of this article at jemds.com.

\section{REFERENCES}

[1] Huang C, Wang Y, Li X, et al. Clinical features of patients infected with 2019 novel coronavirus in Wuhan, China. Lancet 2020;395(10223):497-506.

[2] Tahamtan A, Ardebili A. Real-time RT-PCR in COVID-19 detection: issues affecting the results. Expert Rev Mol Diagn 2020;20(5):453-4.

[3] Sun J, Xiao J, Sun R, et al. Prolonged persistence of SARSCoV-2 RNA in body fluids. Emerg Infect Dis 2020;26(8):1834-8.

[4] Joynt GM, Wu WK. Understanding COVID-19: what does viral RNA load really mean? Lancet Infect Dis 2020;20(6):635-6.

[5] Xiao AT, Tong YX, Gao C, et al. Dynamic profile of RT-PCR findings from 301 COVID-19 patients in Wuhan, China: a descriptive study. J Clin Virol 2020;127:104346.

[6] Ling Y, Xu SB, Lin YX, et al. Persistence and clearance of viral RNA in 2019 novel coronavirus disease rehabilitation patients. Chin Med J (Engl) 2020;133(9):1039-43.

[7] Fu Y, Han P, Zhu R, et al. Risk factors for viral RNA shedding in COVID-19 patients. Eur Respir J 2020;56(1):2001190.

[8] Li TZ, Cao ZH, Chen Y, et al. Duration of SARS-CoV-2 RNA shedding and factors associated with prolonged viral shedding in patients with COVID-19. J Med Virol 2020;93(1):506-12.

[9] Lan L, Xu D, Ye G, et al. Positive RT-PCR test results in patients recovered from COVID-19. JAMA 2020;323(15):1502-3.

[10] Wölfel R, Corman VM, Guggemos W, et al. Virological assessment of hospitalized patients with COVID-2019. Nature 2020;581(7809):465-9.

[11] Guan WJ, Liang WH, Zhao Y, et al. Comorbidity and its impact on 1590 patients with COVID-19 in China: a nationwide analysis. Eur Respir J 2020;55(5):2000547.

[12] Zhou F, Yu T, Du R, et al. Clinical course and risk factors for mortality of adult inpatients with COVID-19 in Wuhan, China: a retrospective cohort study. Lancet 2020;395(10229):1054-62.

[13] Shi D, Wu W, Wang Q, et al. Clinical characteristics and factors associated with long-term viral excretion in patients with severe acute respiratory syndrome coronavirus 2 infection: a single-center 28-day study. J Infect Dis 2020;222(6):910-8. 
[14] Fang L, Karakiulakis G, Roth M. Are patients with hypertension and diabetes mellitus at increased risk for COVID-19 infection? Lancet Respir Med 2020;8(4):e21.

[15] Schiffrin EL, Flack JM, Ito S, et al. Hypertension and COVID-19. Am J Hypertens 2020;33(5):373-4.
[16] Gautret P, Lagier JC, Parola P, et al. Hydroxychloroquine and azithromycin as a treatment of COVID-19: results of an open-label non-randomized clinical trial. Int J Antimicrob Agents 2020;56(1):105949.

[17] Lighter J, Raabe V. azithromycin should not be used to treat COVID-19. Open Forum Infect Dis 2020;7(6):ofaa207. 